\title{
Neumonía asociada a la ventilación mecánica
}

\author{
E. Diaz ${ }^{a, *}$, L. Lorente ${ }^{b}$, J. Valles ${ }^{c}$ y J. Rello ${ }^{d}$
}

\begin{abstract}
${ }^{a}$ Servicio de Medicina Intensiva, Hospital Universitari Joan XXIII Tarragona, Universitat Rovira $i$ Virgili, IISPV, CIBER Enfermedades Respiratorias, CIBERes, Tarragona, España

bUnidad de Cuidados Intensivos (UCI), Hospital Universitario de Canarias, Canarias, España

${ }^{c}$ Coordinador del Servei de Medicina Intensiva, Area de Crítics, Hospital de Sabadell, CIBER de Enfermedades Respiratorias, Barcelona, España

'Servicio de Medicina Intensiva, Vall D'Hebron, UAB, Institut de Recerca Vall D'Hebron,

CIBER de Enfermedades Respiratorias, Barcelona, España
\end{abstract}

Recibido el 4 de marzo de 2010; aceptado el 12 de marzo de 2010

Disponible en Internet el 13 de mayo de 2010

\author{
PALABRAS CLAVE \\ Neumonía asociada a \\ la ventilación \\ mecánica; \\ Tratamiento; \\ Traqueobronquitis
}

\section{KEYWORDS}

Ventilator-associated pneumonia;

Therapy;

Trachobronchitis

\begin{abstract}
Resumen
La neumonía es la segunda complicación infecciosa en frecuencia en el medio hospitalario, y ocupa el primer lugar en los servicios de medicina intensiva. El $80 \%$ de los episodios de neumonía nosocomial se produce en pacientes con vía aérea artifical y se denomina neumonía asociada a la ventilación mecánica (NAV). La NAV es la causa más frecuente de mortalidad entre las infecciones nosocomiales en las $\mathrm{UCl}$, principalmente si son debidas a Pseudomonas aeruginosa y Staphylococcus aureus resistente a meticilina (MRSA). Además, incrementa los días de ventilación mecánica y la estancia media en la UCl y hospitalaria. A pesar de las pruebas disponibles, el diagnóstico de una NAV sigue siendo clínico. La presencia de una opacidad en la radiología de tórax y secreciones traqueales purulentas son condiciones imprescindibles para su diagnóstico. Además, deberemos evaluar su estado y los factores de riesgo para patógenos de difícil tratamiento. Si la NAV es precoz y no existen estos factores de riesgo, la mayoría de las pautas empíricas presentan una cobertura correcta de la flora que nos encontraremos. Sin embargo, si el diagnóstico de NAV se realiza en un paciente con más de una semana de ventilación mecánica, en tratamiento antibiótico, o con factores de riesgo deberemos individualizar la pauta. (c) 2010 Elsevier España, S.L. y SEMICYUC. Todos los derechos reservados.
\end{abstract}

\section{Mechanical ventilation associated pneumonia}

\section{Abstract}

The second most important infectious complication in hospitalized patients is pneumonia, this occupying the first place in the Intensive Care Units (ICU). Approximately $80 \%$ of the episodes of nosocomial pneumonia occur in patients with an artificial airway, which is

\footnotetext{
*Autor para correspondencia.

Correo electrónico: emilio.diaz.santos@gmail.com (E. Diaz).
} 
called ventilation-associated pneumonia (VAP). VAP is the most frequent cause of mortality among nosocomial infections in the ICU, mainly if they are caused by Pseudomonas aeruginosa and Methicillin-resistant Staphylococcus aureus (MRSA). It also increases days of mechanical ventilation and length of stay in the ICU and hospital. In spite of the available diagnostic procedures, the diagnosis of VAP continues to be a clinical one. The presence of X-ray infiltrates and purulent endotracheal secretions are the essential conditions for the diagnosis. We should also evaluate the patient's condition and the risk factors for difficult-to-treat pathogens. If the VAP is early and there are no risk factors, most of the empiric antibiotic strategies will provide correct coverage of the flora found. However, if the diagnosis of VAP is made in a patient who has been receiving mechanical ventilation for more than one week, under antibiotic use, with risk factors for multirresistant pathogens, we should individualize the empiric antibiotic treatment.

(c) 2010 Elsevier España, S.L. and SEMICYUC. All rights reserved.

\section{Introducción}

La neumonía es la segunda complicación infecciosa en frecuencia en el medio hospitalario, y ocupa el primer lugar en los servicios de medicina intensiva, cuyo riesgo está aumentado más de 20 veces por la presencia de la vía aérea artificial ${ }^{1}$. El $80 \%$ de los episodios de neumonía nosocomial se produce en pacientes con vía aérea artificial y se denomina neumonía asociada a la ventilación mecánica $(\mathrm{NAV})^{2}$. La NAV afecta hasta un $50 \%$ de los pacientes, según la patología de ingreso, que ingresan en $\mathrm{UCl}$, y presenta una densidad de incidencia que varía entre 10-20 episodios por cada mil días de ventilación mecánica ${ }^{3}$, con un riesgo diario de entre $1-3 \%^{4}$. Este riesgo es mayor en los primeros días, y es especialmente alto, en pacientes ingresados en coma, donde se puede llegar a diagnosticar hasta en el $50 \%$ de los pacientes $^{5}$. Las enfermedades de base y condiciones que aumentan el riesgo de presentar una NAV se pueden apreciar en la tabla 1.

Tabla 1 Enfermedades, comorbilidades y situaciones que aumentan el riesgo de presentar neumonía asociada a ventilación mecánica

\begin{tabular}{cl}
\hline $\begin{array}{c}\text { Enfermedades y } \\
\text { comorbilidades }\end{array}$ & $\begin{array}{l}\text { Politraumatismo (especialmente } \\
\text { traumatismo craneal) } \\
\text { Presencia de coma o sedación } \\
\text { profunda } \\
\text { Parada cardiorespiratoria }\end{array}$ \\
& Periodo postoperatorio precoz \\
& Quemados con lesión pulmonar por \\
& inhalación \\
& Enfermedad pulmonar obstructiva \\
& crónica u otra enfermedad respiratoria \\
& crónica \\
& Pacientes inmunodeprimidos \\
& Enfermedad grave previa \\
& Contraindicación para la posición de \\
semiincorporado \\
Intubación nasotraqueal \\
Exposición a antibiótico \\
Politransfusión \\
Transporte intrahospitalario
\end{tabular}

La NAV conlleva un aumento en la estancia hospitalaria ${ }^{6}$, con un coste por cada NAV superior a los 40.000 dólares en EE.UU. ${ }^{7}$ y, si se trata tarde $o$ es causada por organismos multiresistentes se ha relacionado con un aumento en la mortalidad $^{8,9}$.

\section{Fisiopatología}

Aunque clásicamente se han venido distinguiendo 4 vías patogénicas para el desarrollo de NAV (aspiración de secreciones colonizadas procedente de la orofaringe, por contigüidad, por vía hematógena, y a través de los circuitos o tubuladuras), la aspiración de secreciones procedentes de la orofaringe es la vía mayoritaria y casi única. La vía aérea inferior es una zona habitualmente estéril en personas sanas, la excepción se limita a pacientes con enfermedades crónicas pulmonares. En los pacientes bajo ventilación mecánica, la intubación endotraqueal, en cambio, rompe el aislamiento de la vía aérea inferior. El neumotaponamiento del tubo endotraqueal es un sistema diseñado para aislar la vía aérea, evitando pérdidas aéreas y la entrada de material a los pulmones, pero no es completamente estanco. Por encima del neumotaponamiento se van acumulando secreciones que, provenientes de la cavidad oral, están contaminadas por los patógenos que colonizan la orofaringe. Estas secreciones contaminadas pasan alrededor del neumotaponamiento y alcanzan la vía aérea inferior. Esta cantidad o inóculo será escaso si existen pocas secreciones acumuladas, pero si la integridad del sistema está alterada, el inóculo que pueda llegar al parénquima pulmonar será mayor. Cuando este inóculo supera la capacidad de defensa del huésped, se produce la reacción inflamatoria cuya expresión histológica es la aparición de infiltrado agudo con leucocitos polimorfonucleares. Externamente, apreciaremos la existencia de secreciones respiratorias, que son aspiradas con sondas de aspiración por dentro del tubo endotraqueal. Se ha comprobado que una baja presión del neumotaponamiento, que permitiría un mayor paso de secreciones, se puede asociar al desarrollo de $\mathrm{NAV}^{10}$. Por otro lado, una presión mayor comprometería la circulación en la mucosa respiratoria pudiendo llegar a lesionarla. Por todo ello, se recomienda que la presión del neumotaponamiento se mantenga entre $25-30 \mathrm{~cm}$ de $\mathrm{H}_{2} \mathrm{O}^{10}$. Así, se entiende que las medidas dirigidas al cuidado de la 
vía aérea evitando la presencia de secreciones, su contaminación, o el paso de ellas a la vía aérea inferior son potenciales objetivos para la prevención de la NAV.

\section{Microbiología}

Los factores que influyen en la etiología de la NAV son el tiempo de ventilación mecánica ${ }^{11}$, la administración previa de antibioticoterapia ${ }^{12}$, además de algunos factores dependientes del huésped como la presencia de EPOC $^{13}$ o coma ${ }^{5}$. Además, hay que destacar que la etiología depende en gran medida de factores locales ${ }^{14,15}$. Así, la etiología difiere entre las diferentes $\mathrm{UCl}$ de diferentes hospitales ${ }^{14}$ e incluso, entre las distintas $\mathrm{UCl}$ de un mismo hospital ${ }^{15}$. La etiología microbiológica más frecuente se resume en la tabla $2^{16,17}$.

Los episodios de NAV se han clasificado desde hace mucho tiempo en NAV precoz y tardía. Esta diferenciación tiene la ventaja de agrupar los microorganismos en 2 grupos de etiología con implicaciones terapéuticas. Los episodios de NAV precoces suelen estar producidos por patógenos como Streptococcus pneumoniae, Haemophilus influenzae y Staphylococcus aureus sensible a meticilina. Estos patógenos no suelen presentar problemas para su tratamiento antibiótico, y la mayoría de las pautas de tratamiento empírico aseguran que serán fármacos activos contra ellos.

En contraste, los pacientes con episodios de NAV tardíos suelen presentar riesgo de que esta infección esté producida por microorganismos con un perfil de resistencia antibiótica diferente. Entre estos se encuentran Pseudomonas aeruginosa, Acinetobacter baumannii, Staphylococcus aureus resistente a meticilina, sobre todo, aunque también pueden existir otros bacilos gramnegativos. Además, la etiología puede estar modulada por la existencia de enfermedades de base (EPOC, inmunodepresión), tratamiento antibiótico previo o factores locales (alta presión de colonización por algún patógeno), favoreciendo, la colonización primero, y posteriormente la presencia de episodios por algunos de los microorganismos de difícil tratamiento.

\section{Clínica y diagnóstico}

La situación clínica ante la que sospecharemos una NAV es un paciente en ventilación mecánica que presenta fiebre y secreciones purulentas por el tubo traqueal. El diagnóstico no ha cambiado, en esencia, en los últimos años. La presencia de una opacidad en la radiología de tórax junto con evidencia de infección local (secreciones purulentas por el tubo endotraqueal), y sistémica (fiebre y/o leucocitosis), nos da el diagnóstico clínico.

Aunque esta definición no aportaría mayores problemas en pacientes no ventilados, el hecho de que haya muchas entidades que cursen con infiltrados radiológicos (síndrome de distrés respiratorio agudo, edema agudo de pulmón, atelectasias, embolismo pulmonar, infiltración neoplásica) en pacientes que puedan ya presentar fiebre y/o leucocitosis por otras razones, complican el diagnóstico.

Otro punto de controversia durante años ha sido el método de diagnóstico etiológico. Una vez realizado el diagnóstico clínico, la recomendación es realizar una prueba de diagnóstico etiológico antes de iniciar o cambiar el tratamiento antibiótico, sin que esto comporte un retraso en el inicio de su administración ${ }^{18}$. Actualmente, en las $\mathrm{UCl}$ europeas los métodos realizados con fibrobroncoscopio no son mayoritarios ${ }^{19}$, realizándose en el $23 \%$ de los pacientes con NAV, mientras que en el $62 \%$ se realiza broncoaspirado traqueal cuantitativo.

La existencia de una vía aérea artificial comporta que la esterilidad de la vía aérea inferior se pierde a las pocas horas de intubar a un paciente ${ }^{20}$. Así, las muestras microbiológicas cualitativas, como el aspirado traqueal simple, casi siempre nos mostraran la existencia de microorganismos sin que ello implique un papel patogénico en la NAV que presente el paciente en ese momento. Por otro lado, el cultivo negativo debe hacer cuestionar el diagnóstico de NAV, sobre todo si no ha habido introducción o cambio de antibiótico recientemente. La realización de muestras mediante fibrobroncoscopio nos permite acceder al tracto respiratorio inferior, y en muestras de buena calidad diagnosticar la etiología de la NAV con mayor seguridad. La realización de cultivos cuantitativos en las muestras microbiológicas extraídas mediante fibrobroncoscopio es común, como habría de ser el examen de la calidad de la muestra. Cuando se realiza la obtención mediante catéter telescopado protegido, una muestra de calidad deberá presentar menos de $1 \%$ de células epiteliales (un número mayor sugeriría colonización orofaríngea) y más de 10 neutrófilos por campo ${ }^{21}$. En el lavado broncoalveolar

Tabla 2 Etiología de neumonía asociada a la ventilación mecánica

\begin{tabular}{llll}
\hline & Kollef $^{17}(\mathrm{n}=398)$ & Agbath $^{16}(\mathrm{n}=313)$ & NASCENT $^{34}(\mathrm{n}=93)$ \\
\hline $\begin{array}{l}\text { Grampositivos } \\
\text { MSSA }\end{array}$ & $35(8,8)$ & $68(21,7)$ & $15(16,1)$ \\
MRSA & $59(14,8)$ & $25(8,0)$ & $10(10,7)$ \\
Streptococcus pneumoniae & & $24(7,7)$ & $6(6,4)$ \\
Otros Streptococcus spp & & $13(4,2)$ & \\
Gramnegativos & $57(14,3)$ & & $19(20,4)$ \\
Pseudomonas aeruginosa & $8(2,0)$ & $43(13,7)$ & $6(6,4)$ \\
Acinetobacter baumannii & $38(9,5)$ & $10(3,2)$ & $15(16,1)$ \\
Enterobacteriaceae & & $64(20,4)$ & $6(6,4)$ \\
Haemophilus influenzae & $52(16,6)$ & \\
\hline
\end{tabular}

MRSA: Staphylococcus aureus resistente a meticilina; MSSA: Staphylococcus aureus sensible a meticilina. 
(LBA) de pacientes con neumonía, la presencia de neutrófilos es un hallazgo constante, aunque no exclusivo, ya que puede encontrarse en otras entidades como el síndrome de distrés respiratorio agudo. En cambio, en broncoaspirados traqueales el número de leucocitos polimorfonucleares tiene menor valor como discriminante en cuanto a presencia o no de neumonía ${ }^{21}$, a diferencia de la valoración de un esputo de buena calidad en pacientes con neumonía comunitaria (menos de 10 células epiteliales y más de 25 leucocitos por campo) ${ }^{22}$, aunque se suele considerar los mismos valores para considerar su calidad ${ }^{23}$.

\section{Tratamiento}

El manejo conlleva 2 tratamientos simultáneos. Por un lado el tratamiento de soporte y por otro, el tratamiento antibiótico. El tratamiento de soporte se inicia con una ventilación mecánica ajustada a las necesidades del paciente. De entrada, un paciente que desarrolla una NAV no está, al menos el primer día en condiciones de ser extubado. Si se estabiliza rápidamente podrá ser reevaluado. La ventilación mecánica irá dirigida a buscar la mejor oxigenación de los tejidos con el menor daño secundario. Para ello, será importante no olvidar que la mejor fracción inspirada de oxígeno $\left(\mathrm{FiO}_{2}\right)$ es la menor $\mathrm{FiO}_{2}$, ya que el oxígeno a altas concentraciones puede ser tóxico. De hecho, en los pacientes con infección pulmonar, $\mathrm{FiO}_{2}$ tan bajas como el $50 \%$ pueden ser tóxicas, aunque faltan estudios que permitan marcar de forma clara el umbral de $\mathrm{FiO}_{2}$ que pueda considerarse tóxico en humanos ${ }^{24}$. La administración de presión positiva al final de la espiración (PEEP) ayuda a mejorar la oxigenación, pero se ha de buscar un balance entre la oxigenación, la no sobredistensión pulmonar y las necesidades del paciente.

Respecto al tratamiento antibiótico, lo más importante es no demorar un tratamiento efectivo ya que el tratamiento empírico inicial inadecuado conlleva una mayor mortali$\mathrm{dad}^{25}$ (fig. 1). Si la NAV es precoz y no existen estos factores de riesgo, la mayoría de las pautas empíricas presentan una cobertura correcta de la flora que nos encontraremos. Sin embargo, si el diagnóstico de NAV se realiza en un paciente con más de una semana de hospitalización, en tratamiento antibiótico, o con factores de riesgo para multiresistentes deberemos individualizar la pauta. Si se realiza una prueba de diagnóstico etiológico y disponemos de la información de la tinción de Gram nos servirá para orientar el tratamiento empírico.

Si no disponemos de esta prueba: si el paciente está colonizado por S. aureus resistente a meticilina (MRSA) o en el área existen varios pacientes colonizados o infectados por

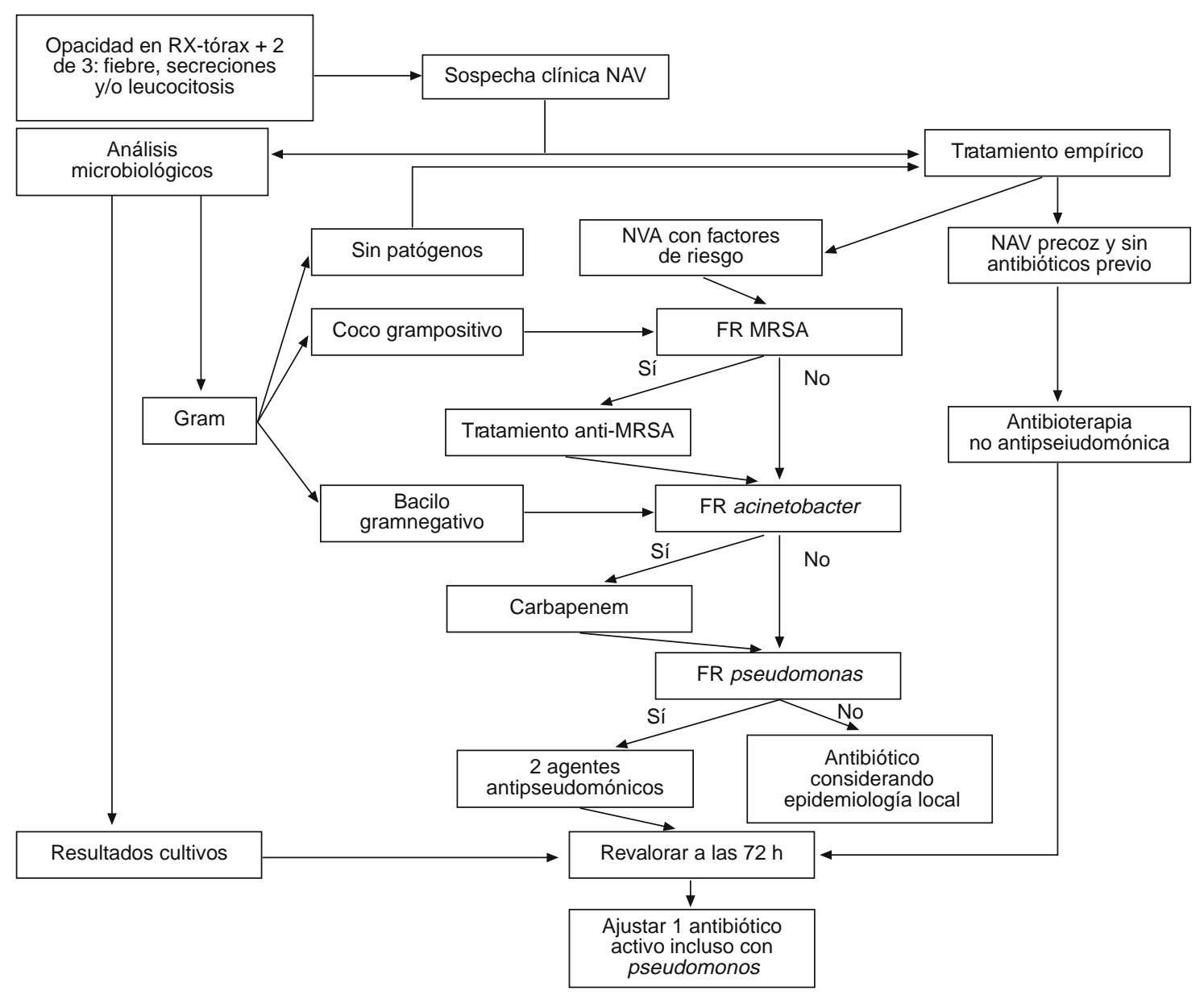

Figura 1 Algoritmo de diagnóstico y tratamiento de neumonía asociada a la ventilación mecánica (modificado de referencia 26). Abreviaciones: FR: factor de riesgo; MRSA: Staphylococcus aureus resistente a meticilina; NAV: neumonía asociada a la ventilación mecánica. 
MRSA, la pauta empírica debe contemplar su tratamiento. $\mathrm{Si}$ no es así, pero existe riesgo de $A$. baumannii, por trabajar en una unidad con una alta densidad de colonización, se debe de tratar empíricamente. Por último, además se ha de considerar que con o sin estos dos patógenos previamente revisados, en las condiciones previas se ha de tener en cuenta que la etiología puede deberse a $P$. aeruginos $a^{26}$.

\section{Prevención}

La prevención se basará principalmente en reducir la colonización orofaríngea, el inóculo 0 ambas $^{27}$. Esta prevención se puede articular en dos grupos de medidas: generales y específicas. Entre las medidas específicas en el cuidado de la vía aérea artificial figura la reducción de la colonización orofaríngea o del inóculo que llegará al tracto respiratorio inferior. El lavado de la cavidad oral con clorhexidina y la descontaminación digestiva intestinal buscan reducir la colonización orofaríngea ${ }^{28}$. La mayoría de prácticas preventivas van encaminadas a la reducción del volumen de secreciones orofaríngeas que llegarán al tracto respiratorio inferior.

Se han elaborado diferentes guías para la prevención de la $\mathrm{NAV}$, y las discrepancias en las recomendaciones pueden deberse fundamentalmente a los estudios elegidos y al desacuerdo con la interpretación de los resultados de los estudios $^{29}$.

Se recomienda la intubación orotraqueal sobre la nasotraqueal, salvo que existan contraindicaciones. La intubación nasotraqueal se ha asociado con mayor riesgo de sinusitis que la orotraqueal, y la sinusitis es un factor de riesgo para el desarrollo de NAV. Se recomienda que la presión del balón de neumotaponamiento se mantenga entre 25-30 $\mathrm{cmH}_{2} \mathrm{O}$ porque presiones menores se han asociado a mayor riesgo de $\mathrm{NAV}^{30}$, y presiones superiores se han asociado a lesiones en la mucosa traqueal ${ }^{10}$.

La utilización de un tubo endotraqueal con una luz para la aspiración de las secreciones subglóticas ha demostrado disminuir la incidencia de NAV de comienzo precoz en pacientes con una duración de la ventilación mecánica mayor de $72 \mathrm{~h}$ en un metaanálisis ${ }^{31}$. En un estudio aleatorizado se objetivó una menor incidencia de NAV de comienzo precoz en el grupo de pacientes ventilados con un tubo endotraqueal que disponía de un balón de pared ultrafina de poliuretano ${ }^{32}$. En un estudio aleatorizado la utilización de un tubo con aspiración de las secreciones subglóticas y balón de pared ultrafina de poliuretano consiguió disminuir la NAV de comienzo precoz y tardío ${ }^{33}$. Por lo tanto, parece lógico asociar aspiración de las secreciones subglóticas y un balón de pared ultrafina. Por otro lado, la utilización de tubos endotraqueales impregnados en plata redujo la incidencia de NAV en un estudio randomizado ${ }^{34}$.

Se recomienda la utilización de protocolos de retirada de la ventilación mecánica, sedación relajación y ventilación mecánica no invasiva para intentar una extubación lo más precoz posible, porque a mayor duración de la ventilación mecánica existe un mayor riesgo de NAV. También se recomienda la realización una traqueostomía precoz en pacientes que se sospeche que vayan a requerir la ventilación mecánica durante un tiempo prolongado porque en un metaanálisis se apreció una reducción de la duración de la ventilación mecánica y la estancia en $\mathrm{UCI}$, aunque no la incidencia de $\mathrm{NAV}^{35}$.

No se recomienda la utilización rutinaria de los filtros respiratorios porque no disminuyen la incidencia de NAV; aunque sería recomendable utilizarlos en pacientes sometidos a ventilación mecánica, con sospecha o confirmación de infecciones pulmonares que se transmiten por gotas (como la tuberculosis pulmonar bacilífera o infecciones por virus) para evitar la contaminación del aire ambiental y prevenir la infección de los trabajadores y familiares. Tampoco es necesario el cambio rutinario de las tubuladuras porque no disminuye el riesgo de NAV.

En un metaanálisis no se han encontrado diferencias en la incidencia de NAV con el uso de un intercambiador de calor y humedad $(\mathrm{ICH})$ o un humidificador de agua caliente $(\mathrm{HAC})$, aunque el coste de la humidificación es menor con el $\mathrm{ICH}^{36}$. Por lo tanto se recomienda el uso de $\mathrm{ICH}$; pero podría utilizarse un $\mathrm{HAC}$ en pacientes que tengan secreciones espesas o atelectasias porque pueden aportar una mayor humidificación. Por otro lado, hay que asegurar el adecuado drenaje del condensado que se puede acumular en las tubuladuras.

En un metaanálisis no se encontraron diferencias en la incidencia de NAV con la utilización de un sistema de aspiración abierto (SAA) o un sistema de aspiración cerrado (SAC), aunque el SAC resultaba más costoso ${ }^{37}$. Por lo tanto, sería suficiente la utilización rutinaria de un SAA, pero sería recomendable utilizar el SAC en pacientes con sospecha o confirmación de infecciones pulmonares que se transmiten por aire o por gotas (como la tuberculosis pulmonar bacilífera o infecciones por virus, por motivos de seguridad para los trabajadores) o en pacientes con inestabilidad hemodinámica gasométrica (por motivos de seguridad para el paciente).

En un metaanálisis se encontró que las camas cinéticas disminuían la incidencia de NAV, sin embargo, no reducían la tasa de mortalidad, duración de la ventilación mecánica, duración de la estancia en $\mathrm{UCl}$, ni la duración de la estancia hospitalaria ${ }^{38}$. Además, la mayoría de los pacientes que estuvieron en camas cinéticas presentaron complicaciones, como la intolerancia a la continua vibración, extubación accidental, retirada accidental de catéteres vasculares, parada cardiaca, arritmias, problemas con las tracciones, incremento de la presión intracraneal ${ }^{38}$. Por lo tanto, no se recomienda la utilización rutinaria de las camas cinéticas.

Se recomienda que los pacientes permanezcan en posición de semiincorporado, porque se ha objetivado una menor aspiración en el interior de las vías aéreas y una menor incidencia de NAV que en la posición en decúbito supino.

En un metaanálisis no se encontraron diferencias significativas en la incidencia de NAV con la utilización de una sonda gástrica o pospilórica para la nutrición artificial ${ }^{39}$. Por lo tanto, no parece necesario el uso rutinario de una sonda pospilórica.

En varios metaanálisis se ha concluido que la descontaminación digestiva selectiva disminuye la incidencia de NAV y de mortalidad. Sin embargo, su aplicación no se ha generalizado debido a motivos como la posible aparición de resistencias antimicrobianas, que su apropiada aplicación es compleja y requiere de una monitorización microbiológica, y 
se cuestiona su relación coste beneficio. Por lo tanto, su administración sigue en controversia.

En un metaanálisis publicado se concluyó que la utilización de la descontaminación oral con clorhexidina disminuía la incidencia de $\mathrm{NAV}^{40}$. Por lo tanto, parece razonable su utilización durante la higiene oral periódica que se realiza en los pacientes en ventilación mecánica.

En un metaanálisis la administración profiláctica de antibióticos por vía respiratoria no disminuyó la incidencia de NAV y existe el posible riesgo de aumento de las resistencias microbianas ${ }^{41}$. Por lo tanto, no se recomienda su utilización de forma rutinaria.

El uso de antibióticos en la prevención de la NAV presenta un papel bimodal. Su uso como profilaxis en pacientes en coma, en dos únicas dosis en el momento de la intubación y $12 \mathrm{~h}$ después, se ha asociado con una reducción de la NAV ${ }^{5}$. Esta medida no conllevaría una selección de patógenos. En cambio, la administración de antibioterapia favorecería una selección de patógenos como MRSA, A. baumannii o P. aeruginosa.

En varias ocasiones se ha comunicado la transmisión de infección cruzada a través del personal sanitario. Es el mecanismo de transmisión habitual en el caso de MRSA o A. baumannii. Por ello, se deberían tomar unas adecuadas medidas de barrera entre cada paciente y desinfectar los equipos y dispositivos respiratorios reutilizables entre pacientes.

Un avance reciente en la prevención de la NAV ha venido de la aplicación de una serie de medidas en el cuidado de los pacientes. Así, Resar et al han demostrado como la implementación de un paquete de medidas (care bundle), puede asociarse a una disminución del número de NAV. En el estudio de Resar et $\mathrm{al}^{42}$ las medidas adoptadas fueron profilaxis de las úlceras de estrés, profilaxis de la trombosis venosa profunda, elevación de la cabecera de la cama, y la retirada diaria de la sedación y valoración de la capacidad de ser extubado o no (sedation vacation). En este estudio multicéntrico, las $\mathrm{UCI}$ que presentaron una adherencia a las medidas presentaron una reducción media del número de NAV del 44,5\%. Este enfoque se ha mostrado también válido para la reducción de la bacteriemia relacionada con el cateterismo venoso central ${ }^{43}$.

El uso de la traqueostomía como forma de prevención de la NAV ha sido evaluado en varios estudios ${ }^{44-47}$. En los 3 primeros estudios se realizó aleatorización entre traqueostomía precoz o tardía con un total de 289 pacientes, su uso no se asoció a una disminución de la NAV [riesgo relativo 0,88 (IC 95\%: 0,70-1,10)]. Más recientemente, Rumbak et al en un estudio aleatorizado en pacientes con expectativa de tiempo de ventilación mecánica superior a 14 días apreciaron una reducción en mortalidad, tiempo en $\mathrm{UCl}$, tiempo en ventilación mecánica y frecuencia acumulada de neumonía ${ }^{47}$. Este estudio, sin embargo, presenta unos resultados controvertidos que no han sido corroborados por otros grupos de investigadores hasta el momento.

Los episodios de NAV posteriores a la realización de la traqueostomía suelen ser debidas a $P$. aeruginos $a^{48}$.

\section{Conclusión}

El diagnóstico de la NAV es fundamentalmente clínico, y se basa en la presencia de secreciones purulentas y en la aparición de infiltrados en la radiología de tórax. Hay que distinguir la traqueobronquitis de la neumonía y la identificación etiológica ayuda a desescalar el tratamiento o reevaluar. El tratamiento antibiótico empírico se deberá basar en las comorbilidades del paciente, el tiempo de hospitalización, el uso previo de antibóticos y, sobre todo, la epidemiología local.

\section{Bibliografía}

1. Haley RW, Hooton TM, Culver DH, Stanley RC, Emori TG, Hardison CD, et al. Nosocomial infections in US hospitals, 197576: Estimated frequency by selected characteristics of patients. Am J Med. 1981;70:947-59.

2. Rello J, Diaz E, Rodríguez A. Advances in the management of pneumonia in the intensive care unit: review of current thinking. Clin Microbiol Infect. 2005;11(suppl 5):30-8.

3. Olaechea PM, Ulibarrena MA, Alvarez-Lerma F, Insausti J, Palomar M, De la Cal MA, ENVIN-UCI Study Group. Factors related to hospital stay among patients with nosocomial infection acquired in the intensive care unit. Infect Control Hosp Epidemiol. 2003;24:207-13.

4. Cook DJ, Walter SD, Cook RJ, Griffith LE, Guyatt GH, Leasa D, et al. Incidence of and risk factors for ventilator-associated pneumonia in critically ill patients. Ann Intern Med. 1998; 129:433.

5. Sirvent JM, Torres A, El-Ebiary M, Castro P, de Batlle J, Bonet A. Protective effect of intravenously administered cefuroxime against nosocomial pneumonia in patients with structural coma. Am J Respir Crit Care Med. 1997;155:1729-34.

6. Kollef MH. What Is Ventilator-Associated Pneumonia and Why Is It Important? Respir Care. 2005;50:714-21.

7. Rello J, Ollendorf DA, Oster G, Vera-Llonch M, Bellm L, Redman $R$, et al. VAP Outcomes Scientific Advisory Group. Epidemiology and outcomes of ventilator-associated pneumonia in a large US database. Chest. 2002;122:2115-21.

8. Chastre J, Fagon JY. Ventilator-associated pneumonia. Am J Respir Crit Care Med. 2002;165:867-903.

9. Olaechea PM. Infecciones bacterianas en el paciente crítico: revisión de los estudios publicados entre 2006 y 2008 . Med Intensiva. 2009;33:196-206.

10. Diaz E, Rodriguez AH, Rello J. Ventilator-Associated Pneumonia: Issues Related to the Artificial Airway. Respir Care. 2005;50: 900-906.

11. Trouillet JL, Chastre J, Vuagnat A, Joly-Guillou ML, Combaux D, Dombret MC, et al. Ventilator-associated pneumonia caused by potentially drug-resistant bacteria. Am J Respi Crit Care Med. 1998; 157:531-9.

12. Rello J, Torres A. Microbial causes of ventilator-associated pneumonia. Semin Respir Infect. 1996;11:24-31.

13. Rello J, Ausina V, Ricart M, Puzo C, Quintana E, Net A, et al. Risk factors for infection by Pseudomonas aeruginosa in patients with ventilator-associated pneumonia. Intensive Care Med. 1994;20:193-8.

14. Rello J, Sa-Borges M, Correa H, Leal SR, Baraibar J. Variations in ethiology of ventilator-associated pneumonia around four treatment sites : implications for antimicrobial prescribing practices. Am J Respir Care Med. 1999;160:608-13.

15. Namias J, Samiian L, Nino D, Shirazi E, O’Neill K, Kett DH, et al. Incidence and susceptibility of pathogenic bacteria vary between ICU within a single hospital: implications for empiric antibiotic strategies. J Trauma. 2000;49:638-45.

16. Agbaht K, Díaz E, Muñoz E, Lisboa T, Gomez F, Depuydt PO, et al. Bacteremia in patients with ventilator-associated pneumonia is associated with increased mortality: a study 
comparing bacteremic vs nonbacteremic ventilator-associated pneumonia. Crit Care Med. 2007;35:2064-70.

17. Kollef MH, Morrow LE, Niederman MS, Leeper KV, Anzueto A, Benz-Scott $\mathrm{L}$, et al. Clinical characteristics and treatment patterns among patients with ventilator-associated pneumonia. Chest. 2006;129:1210-8.

18. Timsit JF. Bronchoalveolar lavage for VAP diagnosis: patients must be sampled before any change of antimicrobial therapy. Intensive Care Med. 2007;33:1690-3.

19. Rello J, Koulenti D, Blot S, Sierra R, Diaz E, de Waele JJ, et al. Oral care practices in intensive care units: a survey of 59 European ICUs. Intensive Care Med. 2007;33:1066-70.

20. Sirvent JM, Torres A, Vidaur L, Armengol J, de Batlle J, Bonet A. Tracheal colonisation within $24 \mathrm{~h}$ of intubation in patients with head trauma: risk factor for developing early-onset ventilatorassociated pneumonia. Intensive Care Med. 2000;26:1369-72.

21. Gallego M, Rello J. Diagnostic testing for ventilador-associated pneumonia. Clin Chest Med. 1999;20:671-9.

22. García-Vázquez E, Marcos MA, Mensa J, de Roux A, Puig J, Font $C$, et al. Assessment of the usefulness of sputum culture for diagnosis of community-acquired pneumonia using the PORT predictive scoring system. Arch Intern Med. 2004;164:1807-11.

23. Lisboa T, Rello J. The simple and the simpler in pneumonia diagnosis. Crit Care. 2007;11:140.

24. Gordo-Vidal F, Calvo-Herranz E, Abella-Álvarez A, SalinasGabiña I. Toxicidad pulmonar por hiperoxia. Med Intensiva. 2010;34:134-8.

25. Ibrahim EH, Ward S, Sherman G, Schaiff R, Fraser VJ, Kollef MH. Experience with a clinical guideline for the treatment of ventilator-associated pneumonia. Crit Care Med. 2001;29: 1109-15.

26. Rello J, Diaz E. Pneumonia in the intensive care unit. Crit Care Med. 2003;31:2544-51.

27. Kollef $\mathrm{MH}$. The prevention of ventilator-associate pneumonia. N Engl J Med. 1999;340:627-34.

28. García-Hierro P, de la Cal MA, van Saene HFK, Silvestri L. Un nuevo ensayo clínico con descontaminación digestiva selectiva. Med Intensiva. 2009;33:297-300.

29. Lorente L, Blot S, Rello J. Evidence on measures for the prevention of ventilator-associated pneumonia. Eur Respir J. 2007;30:1193-207.

30. Rello J, Sonora R, Jubert P, Artigas A, Rue M, Valles J. Pneumonia in intubated patients: role of respiratory airway care. Am J Respir Crit Care Med. 1996;154:111-5.

31. Dezfulian C, Shojania K, Collard HR, Kim HM, Matthay MA, Saint $S$. Subglottic secretion drainage for preventing ventilatorassociated pneumonia: a meta-analysis. Am J Med. 2005;118: 11-18.

32. Poelaert J, Depuydt P, De Wolf A, Van de Velde S, Herck I, Blot S. Polyurethane cuffed endotracheal tubes to prevent early postoperative pneumonia after cardiac surgery: a pilot study. J Thorac Cardiovasc Surg. 2008;135:771-6.

33. Lorente L, Lecuona M, Jimenez A, Mora ML, Sierra A. Influence of an Endotracheal Tube with Polyurethane Cuff and Subglottic Drainage on Pneumonia. Am J Respir Crit Care Med. 2007;176: 1079-1083.
34. Kollef MH, Afessa B, Anzueto A, Veremakis C, Kerr KM, Margolis BD, et al. NASCENT Investigation Group. Silver-coated endotracheal tubes and incidence of ventilator-associated pneumonia: the NASCENT randomized trial. JAMA. 2008;300: 805-813.

35. Griffiths J, Barber VS, Morgan L, Young JD. Systematic review and meta-analysis of studies of the timing of tracheostomy in adult patients undergoing artificial ventilation. BMJ. 2005;330: 1243-1246.

36. Kola A, Eckmanns T, Gastmeier P. Efficacy of heat and moisture exchangers in preventing ventilator-associated pneumonia: meta-analysis of randomized controlled trials. Intensive Care Med. 2005;31:5-11.

37. Jongerden IP, Rovers MM, Grypdonck MH, Bonten MJ. Open and closed endotracheal suction systems in mechanically ventilated intensive care patients: a meta-analysis. Crit Care Med. 2007;35:260-70.

38. Delaney A, Gray H, Laupland KB, Zuege DJ. Kinetic bed therapy to prevent nosocomial pneumonia in mechanically ventilated patients: a systematic review and meta-analysis. Crit Care. 2006;10:R70.

39. Marik PE, Zaloga GP. Gastric versus post-pyloric feeding: a systematic review. Crit Care. 2003;7:R46-51.

40. Chan EY, Ruest A, Meade MO, Cook DJ. Oral decontamination for prevention of pneumonia in mechanically ventilated adults: systematic review and meta-analysis. BMJ. 2007;334: 889.

41. Falagas ME, Siempos II, Bliziotis IA, Michalopoulos A. Administration of antibiotics via the respiratory tract for the prevention of ICU-acquired pneumonia: a meta-analysis of comparative trials. Crit Care. 2006;10:R123.

42. Resar R, Pronovost P, Haraden C, Simmonds T, Rainey T, Nolan T. Using a bundle approach to improve ventilator care processes and reduce ventilator-associated pneumonia. Jt Comm J Qual Patient Saf. 2005;31:243-8.

43. Pronovost P, Needham D, Berenholtz S, Sinopoli D, Chu H, Cosgrove $S$, et al. An intervention to decrease catheter-related bloodstream infections in the ICU. N Engl J Med. 2006;355: 2725-2732.

44. Dunham MC, LaMonica C. Prolonged tracheal intubation in the trauma patient. J Trauma. 1984;24:120-4.

45. Rodriguez JL, Steinberg SM, Luchetti FA, Gibbons KJ, Taheri PA, Flint LM. Early tracheostomy for primary airway management in the surgical care setting. Surgery. 1990;108:655-9.

46. Sugerman HJ, Wolfe L, Pasquale MD, Rogers FB, O'Malley KF, Knudson $M$, et al. Multicenter, randomized, prospective trial of early tracheostomy. J Trauma. 1997;43:741-7.

47. Rumbak MJ, Newton M, Truncale T, Schwartz SW, Adams JW, Hazard PB. A prospective, randomized, study comparing early percutaneous dilational tracheotomy to prolonged translaryngeal intubation (delayed tracheotomy) in critically ill medical patients. Crit Care Med. 2004;32:1689-94.

48. Rello J, Lorente C, Díaz E, Bodí M, Boqué C, Sandiumenge A, et al. Incidence, etiology and outcome of nosocomial pneumonia in ventilated patients requiring tracheotomy. Chest. 2003;124:2239-43. 